\title{
BMJ Open GPs' perspectives on managing the NHS Health Check in primary care: a qualitative evaluation of implementation in one area of England
}

\author{
Rachel L Shaw, Helen Lowe, Carol Holland, Helen Pattison, Richard Cooke
}

To cite: Shaw RL, Lowe $\mathrm{H}$, Holland C, et al. GPs' perspectives on managing the NHS Health Check in primary care: a qualitative evaluation of implementation in one area of England. BMJ Open 2016;6:e010951. doi:10.1136/bmjopen-2015010951

- Prepublication history for this paper is available online. To view these files please visit the journal online (http://dx.doi.org/10.1136/ bmjopen-2015-010951).

Received 23 December 2015 Revised 10 March 2016 Accepted 28 April 2016

CrossMark

School of Life \& Health Sciences, Aston University, Birmingham, UK

Correspondence to Dr Rachel L Shaw; r.l.shaw@aston.ac.uk

\section{ABSTRACT}

Objectives: To evaluate the implementation of the National Health Service (NHS) Health Check programme in one area of England from the perspective of general practitioners (GPS).

Design: A qualitative exploratory study was conducted with GPs and other healthcare professionals involved in delivering the NHS Health Check and with patients. This paper reports the experience of GPs and focuses on the management of the Heath Check programme in primary care.

Setting: Primary care surgeries in the Heart of Birmingham region (now under the auspices of the Birmingham Cross City Clinical Commissioning Group) were invited to take part in the larger scale evaluation. This study focuses on a subset of those surgeries whose GPs were willing to participate.

Participants: 9 GPs from different practices volunteered. GPs served an ethnically diverse region with areas of socioeconomic deprivation. Ethnicities of participant GPs included South Asian, South Asian British, white, black British and Chinese.

Methods: Individual semistructured interviews were conducted with GPs face to face or via telephone. Thematic analysis was used to analyse verbatim transcripts.

Results: Themes were generated which represent GPS' experiences of managing the NHS Health Check: primary care as a commercial enterprise; 'buy in' to concordance in preventive healthcare; following protocol and support provision. These themes represent the key issues raised by GPs. They reveal variability in the implementation of NHS Health Checks. GPs also need support in allocating resources to the Health Check including training on how to conduct checks in a concordant (or collaborative) way.

Conclusions: The variability observed in this smallscale evaluation corroborates existing findings suggesting a need for more standardisation. Further large-scale research is needed to determine how that could be achieved. Work needs to be done to further develop a concordant approach to lifestyle advice which involves tailored individual goal setting rather than a paternalistic advice-giving model.

\section{Strengths and limitations of this study}

- General practitioners (GPs) spoke candidly about the challenges of managing the National Health Service (NHS) Health Check which enabled a thorough analysis of implementation issues from grassroots. More research is needed though to further explore the financial incentives to running the Health Check and its potential associations with Quality Outcomes Framework (QOF).

- This was a small sample in one geographic area of England. Nevertheless, the variability of implementation observed in the region evaluated corroborates findings elsewhere further adding to the evidence that more standardisation is required.

- We found that GPs did not always adopt a concordant approach which is required to tailor lifestyle changes to individual patients. It may be necessary for future training to emphasise this, but more research is needed to determine whether this is the case in other areas as well as that evaluated here.

The UK National Health Service (NHS) Health Check was launched in England and Wales in 2009 as a case-finding programme to identify risk levels for cardiovascular disease, diabetes, stroke and recently dementia has been added, in order to reduce the incidence of these lifestyle-related conditions through biomedical measures and behavioural interventions. ${ }^{1}$ The programme is funded through locally enhanced contracts with local government. It aims to reduce future healthcare demand by reducing the risk of developing these conditions and by reducing the complications associated with them. ${ }^{2}$ It is offered to all patients aged $40-74$ every 5 years and produces a 10-year risk score and personal behaviour change development plan. ${ }^{3}$ Biomedical measures include cholesterol, blood glucose, blood pressure and body mass index (BMI). The check is 
scheduled for $20 \mathrm{~min}$, and within that time, patients are asked questions about their health behaviours, for example, smoking, drinking, physical activity, diet and lifestyle goals are set if a patient is judged at risk. Within the session, referrals to additional services are made if appropriate, for example, to smoking cessation counselling, weight loss programmes and alcohol support.

This paper reports the findings from one element of a larger scale evaluation of the implementation of the NHS Health Check in one ethnically diverse and socioeconomically deprived urban area of England. ${ }^{4}$ In the larger study, we found issues relating to a lack of fidelity to intervention protocol especially related to the behavioural interventions or 'lifestyle advice'. Problems were also reported regarding referral procedures; there was inconsistency in use of referral services and methods for recording referral pathways, and confusion over follow-up appointments for the Health Check itself.

Other research evidence has identified patient perceptions of lifestyle-related conditions problematic in terms of conveying a preventive health message ${ }^{5}{ }^{6}$ attendance at a Health Check can be low and risk assessments misinterpreted or judged insignificant by patients; ${ }^{7}$ and the intended emphasis on behaviour change has been lost as patients perceive it as a clinical test and as reassurance of good health which therefore requires no action by them. ${ }^{8}$ UK-based research focusing on the NHS Health Check specifically has identified variability in implementation as a key issue; there have been claims of a postcode lottery in relation to allocated budgets, payments to service providers, tools used to measure risk, and the availability of preventive and lifestyle support services. ${ }^{9} 10$ Patients have expressed the need for individualised services to help them maintain lifestyle changes, and there have been calls for more consistency in explaining the purpose of the NHS Health Check. ${ }^{11}$ From the perspective of general practitioners (GPs) and practice managers, barriers to implementation have included software problems, time pressure and the impact of an increased nursing workload on costs and other services. ${ }^{12}$ To further investigate the management of the NHS Health Check in primary care, this study focused on examining the views of GPs.

\section{METHODS}

Recruitment to the evaluation was undertaken by the local NHS Trust. Initial consent to be contacted was taken by the Trust and those who agreed were then approached by the research team, described as a team of health psychologists contracted by the Trust to evaluate the Health Check. Semistructured individual interviews were conducted with GPs either in person or by telephone to suit GPs' availability. Questions focused on the organisation of the Health Check, recruitment of patients to the programme, specifics of how it was delivered and perceived impact of the Health Check on patient health behaviours. Questions included: Can you tell me about how you organised the Health Check at your practice? What strategies did you employ to recruit patients to the programme? What do you think will be the impact of the NHS Health Check on your patients? Interviews were carried out by RC, a health psychologist, and a research assistant with health psychology training. They lasted between 30 and $60 \mathrm{~min}$. Audio-recordings were transcribed verbatim and transcripts analysed using inductive thematic analysis which focuses on what the experiences of participants and attempts to identify commonalities and differences in their accounts to generate a detailed description of the implementation of the NHS Health Check. ${ }^{13}$ The analysis was led by RLS and discussed within the research team. Initial themes were developed independently by RLS, RC and HL. Themes were then developed through discussion and further independent interpretative work until consensus was reached.

\section{RESULTS}

Nine GPs took part from different GP practices, eight of whom were men. Two identified as South Asian, two as South Asian-British, two white, one black British and one Chinese (see table 1). All except one practice were in socioeconomically deprived areas of Birmingham.

Four themes were generated which will be presented in turn with data extracts to illustrate their significance: primary care as a commercial enterprise, 'buy in' to concordance in preventive healthcare; following protocol and support provision.

\section{PRIMARY CARE AS A COMIMERCIAL ENTERPRISE}

Since the Quality Outcomes Framework (QOF) was introduced in primary care, GP practices have worked within a financial model which provides remuneration for the provision of good quality care and for entering patients onto the diabetes register, for example. Similarly, GPs were financially incentivised to recruit patients to the NHS Health Check programme through the locally enhanced services. This meant that for some, financial issues featured strongly in their decision to offer the NHS Health Check.

\begin{tabular}{|c|c|c|}
\hline Participant number & Sex & Ethnicity \\
\hline GP1 & Male & South Asian-British \\
\hline GP2 & Male & South Asian \\
\hline GP3 & Male & South Asian-British \\
\hline GP4 & Male & Black British \\
\hline GP5 & Female & South Asian \\
\hline GP6 & Male & South Asian \\
\hline GP7 & Male & White \\
\hline GP8 & Male & Chinese \\
\hline GP9 & Male & White \\
\hline
\end{tabular}


'In order to get good payments we had to reach $50 \%$ target within three months otherwise, if you delayed it, remuneration wasn't that good...it was important for us to get the targets very very quickly.' (GP8)

'Some doctors won't do it because they think it's a lot of work to be done for the amount of finance that they will be reimbursed and they are just financially driven whereas other doctors, and I hope I'm one of them, are actually bothered about their patients and actually care.' (GP1)

Most of the GPs interviewed discussed the financial context of delivering the Health Check programme because it was fundamental to their practice as a whole. GP practices function as a commercial enterprise and thus take seriously the financial elements of any service they deliver. However, the second extract from GP1 conveys frustration at needing to prioritise financial reward in a practice that should be focused on caring for patients. This frustration is confounded when GPs perceive that government departments that set targets are not apprised sufficiently in how things work on the ground and what other issues need to be addressed.

'I mean the biggest problem with the screening programme has been I think because it's target driven.... and the people who set these targets don't understand how the practice works.' (GP2)

Surgeries had to develop methods to maximise recruitment and reach targets for remuneration through the locally enhanced service agreements to make running the NHS Health Check financially viable. This was more or less challenging for different practices depending on their size and staffing levels. In some cases, this meant blocking out appointment times, employing new staff and actively seeking out eligible patients by carrying out reviews of notes. This was time intensive and put surgeries under additional pressure.

'What we have had to do is um block some appointments off for healthcare assistants so when people are in the surgery they could be fitted in'. (GP3)

In order to meet targets, some GPs said they screened patients opportunistically when they came to surgery for other reasons and conducted the Health Check there and then. Most GPs we spoke to took up a coordinating role and it was healthcare assistants and practice nurses who carried out the checks, but not exclusively. This was a more efficient use of time and financial resource, although some expressed concern about not being fully aware of how their patients were managed.

\section{'BUY IN' TO CONCORDANCE IN PREVENTIVE HEALTHCARE}

Concordance is the process by which GPs work with patients to reach agreement about treatment; it involves GPs understanding patients' views and patients making sense of the importance of treatment and consequences of (not) taking it. ${ }^{14}$ Challenges to concordance are arguably multiplied when working in preventive healthcare. Nevertheless, among GPs interviewed, there was support for the NHS Health Check based on strong feelings about preventive healthcare. This evaluation took place in an area with a high proportion of South Asian patients, and so issues relating to diabetes were particularly persuasive in terms of supporting the need for the check.

'I think it's a very good idea. We have a very high proportion of our patients who suffer with diabetes, almost $10 \%$ of our patients are diabetic so I thought this was an excellent opportunity to screen those earlier and pick them up and then you know be able to do something about it, you know, lifestyle management... The money was also very useful. I'm not going to lie. That was an added bonus.' (GP5)

The GP above was persuaded because of the potential to help patients prevent conditions like diabetes by buying into preventive health rhetoric. There was also an underlying motivation in the financial reward the practice would receive for participating in this programme. Another GP stressed how important it was for Health Checks to be available through the NHS rather than losing patients to the private sector.

'I think there should be an emphasis on preventing the 'deadly trio', diabetes, kidney disease and cardiovascular disease for middle aged patients. And I think a lot of patients expect this. A lot of them to go to the private sector for them and I think it's good that the NHS should be doing it.' (GP9)

A key aspect of the NHS Health Check, as designed by the Department of Health following the National Institute for Health and Care Excellence (NICE) guidelines, is the focus on lifestyle. This meant that when risks were identified in the biomedical tests, attempts were made to address lifestyle factors. In the area evaluated here, this was to be done using a goal-setting sheet to be worked through with patients to develop a set of lifestyle change goals that is unique to them. The idea here was that this was a collaborative exercise which mirrored a concordant approach to healthcare, that is, an approach where patient and healthcare professional negotiate issues, discussions are equitable and patients are able to make active informed choices. In some cases, this approach was not always taken, as we have reported elsewhere, ${ }^{4}$ and instead the focus was on giving advice. Language like that in the extract below, betrays such a paternalistic approach.

'Personally, I don't give them goals. The practice nurse will follow them up. Whether she sets a target I'm not sure about that.' (GP9) 
This is almost the opposite of what was intended; instead of working together with patients to set goals which suit them, their family, their situation, this suggests a traditional approach where healthcare consultations are purely didactic. Some GPs were aware of this issue and knew the onus was on the patient to make their own lifestyle changes; however, there was some doubt about whether patients would be capable or willing to make such changes.

\begin{abstract}
'Young Asian men...they've got a 60\% high risk, they walk in and I say, 'look you're a man, you're Asian, and you've got family history, you can't change your sex right, you can't change your race, you can't change your family, you've already got three risk factors already without you doing anything which is why you need to be aggressive in your lifestyle change...you're doomed'...that may work quite well, but it may not work quite well...they may say 'oh yeah I need to do something because I'm already kind of in the negative' but then you get some people who say 'you know what's the point because I'm already at risk anyway'.' (GP1)
\end{abstract}

While this GP admits this approach works for some, it does not for others. Clearly, there is a need to establish what else would work. Conducting a consultation in a concordant manner (ie, collaboratively involving the negotiation of treatment and active decision-making on the patient's behalf) requires rapport and a focus on the patient's unique situation. That does not mean the healthcare professional conducting the check needs to know all the patients; it means healthcare professionals need the skills to elicit information from patients which will help them identify which approach will work best with them. Focusing on setting goals that are tailored to each individual is one way of working out what is important to them and what will therefore motivate change.

\section{FOLLOWING PROTOCOL}

To run the NHS Health Check according to protocol required equipment and training in the approach to be taken with patients. The focus was on collaborative healthcare and setting lifestyle goals tailored to individual patients if they were found to have elevated risk in the biomedical tests. There were problems when the programme first started with equipment.

'When it first started...the training wasn't officially organised until a bit later...the support materials didn't arrive at the start, they arrived later on, we got a big box delivered.' (GP3)

Practices participating in the programme were required to meet a particular technology specification to enable them to manage patient recruitment and results data to ensure the checks conducted were recorded. Some GPs used this software to opportunistically screen patients.

'When a patient comes in an alert will come up saying this person needs cardiovascular screening and there's a template so while the patient's there I'll say, 'look you know what, you're over the age of $40^{\prime}$ I'll say, 'you're at risk of having a heart attack in the next 10 years and hence I'm going to do some blood tests, ask you a few questions to alleviate that risk.' (GP1)

There are a number of issues here. Opportunistic screening may not always elicit informed consent; a patient may feel unable to decline their GP even if they are not clear what the tests are for or understand what the results will mean. This description is likely to be a shorthand for a longer explanation; however, it suggests a focus on the biomedical tests with no mention that risk may be reduced by changes in lifestyle. Relatedly, asking questions will not alleviate risk. This would be confusing for a patient making informed consent unlikely.

Developing lifestyle goals was encouraged in the area evaluated by using goal-setting sheets. We asked GPs whether they carried out this aspect of the check alongside the biomedical tests.

'Some people use it more than others but I personally did use some but it depended on which person they ended up seeing because we did have training, myself our practice nurse and the healthcare assistant had the training for that, but not everyone had training for that aspect of things. But then we talked about it in our practice meetings and we conveyed the message to others, not everyone did it as formally as that.' (GP3)

In one practice, there was variation in how the check was delivered. The way this GP described the use and non-use of the goal-setting chart intimates a lack of understanding in the rationale for doing this part of the check. There was some inconsistency in training received and clearly a lack of investment in the activity.

\section{SUPPORT PROVISION}

In order to run the NHS Health Check effectively, it must be followed up by lifestyle support in the shape of services that can help patients maintain the changes they proposed and reach their goals. In some cases, practices employed their own healthcare assistants to deliver these support services, for example, in smoking cessation counselling, weight loss and activity programmes.

\footnotetext{
'The bulk of it is done by the healthcare assistant. Mainly it's weight loss and exercise and diet advice. They may be filtered through to the practice nurse, she does most of that sort of thing.' (GP9)
}

The tone in this extract implies a lack of significance on this element of the Health Check. The issue is not who delivers this support, but whether it is prioritised as a key part of the process; it is not simply about identifying risk but also reducing it through lifestyle change. This is not simply an issue at the level of the practice 
though. There is a lack of well-funded support services in the wider community as well as a lack of knowledge about what is available.

\begin{abstract}
'We have regular contact with the 'Be Active' person. He comes and sees us and says 'I'm not getting enough across'...I would say we've seen him probably a minimum of three months...I know we used to have sessions fairly regularly.' (GP7)
\end{abstract}

It is not only inconsistency of services which prevents GPs from maintaining established links with lifestyle support services, but also lack of financial resource. Previously in the area evaluated GPs were able to prescribe to the free gym at off-peak times, which meant accessing it was challenging for people in this area who often worked long hours. Coupled with cuts in local authority leisure services, this means there is a large proportion of patients who cannot access gyms or cannot afford gym membership.

'What the government needs to do is to supplement some money....and say to the gyms we'll give you this much money but what we want as part of that is we want you to accept patients from inn city practices like this.' (GP1)

This also raises the issue of what constitutes physical activity. Despite government recommendations that walking is a good form of physical activity available to the majority of the population, there was little mention of this as a possible way of making a lifestyle change. Perhaps there is some work to be done with GPs as well as patients regarding the benefits of walking and stair climbing, for example.

Managing high-risk levels of alcohol consumption was challenging for some GPs and staff delivering the Health Check. One GP was concerned that patients would not be honest about alcohol consumption to GPs because often they came from the same religious background and there was a stigma to be managed.

\footnotetext{
'It's mainly an Islam religion practised in this area and the main population are Muslims. I think they'd probably be a bit more open to my nurses about drinking because they are not necessarily Muslims so they're a bit more open to them.' (GP5)
}

Contrary to prior assumptions about the benefits of healthcare professionals being from the same communities as their patients, this GP raises the possibility that difference may be beneficial in this context. Linked to that, one GP described an anonymous counselling service for patients concerned about their alcohol consumption.

'It's a service we run here that is anonymous. They come in and see one particular counsellor. It's all alcohol based but the onus is on the patient, we do it upstairs. We are lucky in that we have a room the counsellor can use, in other practices they may not have it. So, we actually get patients from other practices coming to see the counsellor here.' (GP4)

As the extract suggests, this was an unusual case. On top of the availability of drug and alcohol services though is the potential stigma associated with them especially in communities like this one. These data suggest that for the NHS Health Check to fulfil its remit of offering lifestyle support to reduce risk, some investment is required now to ensure associated savings are made in terms of healthcare costs for people diagnosed with diabetes, cardiovascular disease, stroke and dementia.

\section{DISCUSSION}

The GPs' perspectives presented here reveal variation in how the NHS Health Check is delivered in practice which supports previous findings. ${ }^{4-12} 1516$ Differences include staffing, facilities and fidelity to protocol. Some practices employed healthcare assistants and nurses to deliver the check. Some GPs were actively involved in delivering the check while others oversaw the process and made systemic decisions about how it would be implemented. Varying levels of commitment to the concordant ethos of working together to set lifestyle change goals was evident in GPs' reports. In terms of the provision of lifestyle support, some practices were able to deliver this in-house but others were dependent on other local services which were not always reliable or accessible to their patients. Underlying the implementation of the NHS Health Check was the financial reward if recruitment targets were met. This was reasonable motivation for some GPs, but for others, that financial reward was necessary for them to be able to function as a commercial enterprise. Having said that, there was some unease about the emphasis given to finances for a preventive programme that is fundamental to public health and promises cost-savings for the NHS in the future. Indeed, the potentially complex link between this case-finding programme, the possibility of overdiagnosis and associated cost burden, and its relationship to disease registers and QOF requires further investigation to determine cost-effectiveness.

Since this evaluation of the NHS Health Check in this region, a non-randomised controlled study found no significant differences between UK GP practices providing the check and control practices in prevalence of diabetes, hypertension, coronary heart disease, chronic kidney disease and atrial fibrillation. ${ }^{2}$ Reasons for this remain unclear because the research has not been done. However, our previous analysis ${ }^{4}$ of this small-scale qualitative evaluation suggests that the lack of emphasis placed on the lifestyle elements of the NHS Health Check may be a contributory factor. Furthermore, the significance of the concordant, or collaborative, consultation style has not been fully understood; engaging patients in setting their own goals in a collaborative way will give them ownership over their lifestyle change plan 
and it will ensure it fits with their own circumstance thus making it more achievable. ${ }^{17}$ Further research is required on a large scale since the foundation of Clinical Commissioning Groups to evaluate the delivery of the NHS Health Check against the protocol to identify problem areas and also to identify good practice which can then be translated across the programme nationally. The findings from this study need to be assessed in relation to the design and scale of the study; it was an exploratory study with a small number of GPs in one area of England. However, our findings do support those in other areas which also found variability in implementation. ${ }^{8-12}$ There is a clear need for a greater level of standardisation, but at the same time a certain level of flexibility is required to enable the NHS Health Check to be adapted to fit different demographic groups across the UK. Consequently, more context-specific evaluations within the UK would help determine aspects of the NHS Health Check delivery that are applicable regardless of location and which need to be adapted to be acceptable and feasible within particular settings. Another limitation of this research is that we cannot yet draw conclusions about the relationship between the successful implementation of the NHS Health Check and its potential impact on QOF.

\section{CONCLUSIONS}

This small-scale evaluation has identified variability in how the NHS Health Check is delivered in the area evaluated. The significance of the lifestyle element of the check is not fully understood and lifestyle support provision was limited or not accessible. Further larger scale studies are required to identify good practice that could help the NHS Health Check achieve its laudable aim of reducing risk associated with lifestyle-related conditions including heart disease, diabetes, stroke, kidney disease, atrial fibrillation and dementia.

Acknowledgements The authors acknowledge significant contribution from Jamie Waterall and Mary Rutledge for support in recruitment and management of the project and Yvonne Cooper who helped with data collection. The authors also thank the busy general practitioners who gave their time to data generation for this study.

Contributors RC managed the project as principal investigator, contributed to data collection and analysis, and writing the manuscript; $\mathrm{HL}$ contributed to data analysis and writing the manuscript; $\mathrm{CH}$ and HP supported the research team during data collection and analysis, and contributed to writing the manuscript; RLS contributed to project management and supervision of research staff, led data analysis and writing of the manuscript.

Funding The authors would like to thank Heart of Birmingham Teaching Primary Care Trust (now subsumed under Public Health England and Birmingham Cross City Clinical Commissioning Group) who provided the funding for this work.

Competing interests None declared.
Ethics approval Ethical approval was granted by West Midlands REC (10\H1208159) to recruit GPs from GP surgeries participating in the larger scale evaluation during 2010-2011.

Provenance and peer review Not commissioned; externally peer reviewed.

Data sharing statement Anonymised transcripts are stored securely on site and may be available to researchers on request until the authors outlive the NHS data storage agreement and deletion of data.

Open Access This is an Open Access article distributed in accordance with the Creative Commons Attribution Non Commercial (CC BY-NC 4.0) license, which permits others to distribute, remix, adapt, build upon this work noncommercially, and license their derivative works on different terms, provided the original work is properly cited and the use is non-commercial. See: http://creativecommons.org/licenses/by-nc/4.0/

\section{REFERENCES}

1. NHS Health Check. Free NHS Health Check: helping you prevent heart disease, stroke, diabetes, kidney disease and dementia. 2015. http://www.healthcheck.nhs.uk/commissioners and healthcare professionals/national_nhs_health_check_events/nhs_health check_conference_26th_feb_2015_/\# (accessed 23 Oct 2015).

2. Caley $M$, Chohan $\mathrm{P}$, Hooper J. The impact of NHS Health Checks on the prevalence of disease in general practices: a controlled study. Br J Gen Pract 2014;64:e516-21.

3. Hippisley-Cox J, Coupland C, Vinogradova Y, et al. Derivation and validation of QRISK, a new cardiovascular disease risk score for the United Kingdom: prospective open cohort study. BMJ 2007;335:136.

4. Shaw RL, Pattison HM, Holland C, et al. Be SMART: examining the experience of implementing the NHS Health Check in UK primary care. BMC Fam Pract 2015;16:1

5. Troughton J, Jarvis J, Skinner C, et al. Waiting for diabetes: perceptions of people with pre-diabetes: a qualitative study. Patient Educ Couns 2008;72:88-93.

6. Burgess C, Wright AJ, Forster AS, et al. Influences on individuals' decisions to take up the offer of a health check: a qualitative study. Health Expect 2015;18:2437-48.

7. Eborall H, Davies R, Kinmonth AL, et al. Patients' experiences of screening for type 2 diabetes: prospective qualitative study embedded in the ADDITION (Cambridge) randomised controlled trial. BMJ 2007;335:490.

8. Chipchase L, Waterall J, Hill P. Understanding how the NHS Health Check works in practice. Practice Nursing 2013;24:24-9.

9. Graley CEM, May KF, McCoy DC. Postcode lotteries in public health -the NHS health checks programme in North West London. BMC Public Health 2011;11:738.

10. Krska J, duPlessis R, Chellaswamy $\mathrm{H}$. Implementation of $\mathrm{NHS}$ Health Checks in general practice: variation in delivery between practices and practitioners. Prim Health Care Res Dev 2016;17:385-92.

11. Ismail $\mathrm{H}$, Atkin $\mathrm{K}$. The NHS Health Check programme: insights from a qualitative study of patients. Health Expect 2016;19:345-55.

12. Krska J, duPlessis $\mathrm{R}$, Chellaswamy $\mathrm{H}$. Views of practice managers and general practitioners on implementing NHS Health Checks. Prim Health Care Res Dev 2016;17:198-205.

13. Braun V, Clarke V. Using thematic analysis in psychology. Qual Res Psychol 2006;3:77-101

14. Elwyn G, Edwards A, Britten N. "Doing prescribing": how doctors can be more effective. BMJ 2003;327:864.

15. Holland C, Cooper $Y$, Shaw R, et al. Effectiveness and uptake of screening programmes for coronary heart disease and diabetes: a realist review of design components used in interventions. BMJ Open 2013;3:e003428.

16. Ellis N, Gidlow $C$, Cowap $L$, et al. A qualitative investigation of non-response in NHS health checks. Arch Public Health 2015;73:14

17. Armitage CJ. Effectiveness of experimenter-provided and self-generated implementation intentions to reduce alcohol consumption in a sample of the general population: a randomized exploratory trial. Health Psychol 2009;28:545-53. 